\title{
Sorbus domestica L. (Rosaceae) en Extremadura (España)
}

\section{Yonatan Cáceres Escudero}

Grupo de Investigación Forestal (GIF), Centro Universitario de Plasencia, Universidad de Extremadura (España)

\section{Correspondencia}

Y. Cáceres Escudero

e-mail:ycaceres@unex.es

Recibido: 4 enero 2019

Aceptado: 27 noviembre 2019

Publicado on-line: 1 junio 2020

Editado por: A.V. Pérez-Latorre

\begin{abstract}
Resumen
Este artículo se centra en el estudio de la ecología, biología reproductiva y dinámica poblacional del serbal común (Sorbus domestica L.), especie de escasa distribución en Extremadura. Los resultados muestran que la especie sólo está presente en el macizo montañoso de las Villuercas, en formaciones forestales bien conservadas. Actualmente en Extremadura existen 23 ejemplares repartidos en 5 núcleos poblacionales. No se ha identificado reclutamiento vegetativo o sexual alguno. Consecuentemente, la especie está seriamente amenazada en esta región. Por lo tanto, se requiere la implementación de medidas urgentes de conservación para preservar las poblaciones de $S$. domestica.
\end{abstract}

Palabras clave: Flora, corología, Sorbus domestica, Villuercas.

Abstract

Sorbus domestica L. (Rosaceae) in Extremadura (Spain)

This research is focused on the study of the ecology, reproductive biology and population dynamic of service tree or sorb tree (Sorbus domestica L.), a rare species in Extremadura. The results showed that this species only appears in Villuercas, mainly in well-preserved forests. The total number of individuals currently in Extremadura is of 23 , which are distributed into 5 isolated populations. Both sexual and vegetative regeneration were not identified. Consequently, the species in this region is seriously threatened. Thus, the implementation of urgent measures of conservation are required to preserve $S$. domestica populations.

Key words: Flora, chorology, Sorbus domestica, Villuercas
El género Sorbus pertenece a la familia de las rosáceas, subfamilia Maloidae, e incluye numerosas especies caducifolias $e$ inermes de árboles y arbustos. En base a Flora ibérica (Aedo \& Aldasoro, 1998), en la península lbérica el género Sorbus está representado por nueve especies, de las que cinco están presentes en Extremadura. Junto con Sorbus aria (L.) Crantz, S. domestica L. (syn. Cormus domestica (L.) Spach.) es la especie que presenta menor distribución en dicha comunidad (Calleja \& Cáceres, 2004; Pulido et al., 2007).

Desde antiguo, existe controversia en la delimitación del género Sorbus, que hoy en día sigue sin resolverse (Aedo \& Aldasoro, 1998). Esto se debe a que dicho género muestra una considerable complejidad morfológica, con un gran número de especies apomícticas e híbridos (Oria de Rueda et al., 2006). En base a esta problemática, algunos autores han subdividido Sorbus en 5 géneros diferentes, adscribiendo dentro del género Cormus a S. domestica (Phipps et al., 1990). Ante la falta de consenso, nosotros en este trabajo hemos seguido la nomenclatura utilizada por Flora ibérica (Aedo \& Aldasoro, 1998). Asimismo, todos los taxones nombrados en este trabajo han seguido este tratado.

S. domestica es un árbol caducifolio de hasta 15 metros de altura que presenta un amplio rango geográfico, englobando el centro y sur de Europa y apareciendo de forma muy puntual en el noroeste de África (Figura 1A). En la península Ibérica, dicho taxón muestra una distribución dispersa en el seno de matorrales o a lo largo de márgenes y claros de bosques, generalmente sobre litologías básicas (Oria de Rueda et al., 2006), por lo que se localiza principalmente en la mitad oriental peninsular (Figura 1A), donde estos sustratos son mayoritarios. A pesar de ello, no rehúye los suelos silíceos, siempre que presenten cierto contenido en bases (Oria de Rueda et al., 2006). En Extremadura, la especie ha sido citada únicamente en la provincia de Cáceres (Ladero et al., 1993; Devesa, 1995; Oria de Rueda et al., 2006; Pulido et al., 2007), donde ha sido discutida su naturalidad. Sin embargo, se conoce muy poco sobre la ecología, la dinámica poblacional, la biología reproductiva y el estado de conservación de la especie en dicha comunidad. Para subsanar esta carencia, este estudio 
inicialmente realizó una revisión bibliográfica pormenorizada de la literatura florística y corológica de $S$. domestica en Extremadura, contrastándola y visitando posteriormente los emplazamientos. Paralelamente, se fueron prospectando nuevas áreas para detectar nuevos ejemplares. En todas las poblaciones identificadas, se registraron datos geográficos, bioclimáticos, edáficos, litológicos y florísticos, así como las principales amenazas detectadas y el estado reproductivo de todos los ejemplares de $S$. domestica. Todos los rodales estudiados han sido herborizados. Dichos materiales han sido depositados en el herbario UNEX (Universidad de Extremadura).

\section{Sorbus domestica $\mathrm{L}$.}

ESPAÑA, Cáceres: Robledollano, Umbría de la Venta [30STJ8787], 655-700 m, 22.05.2016, Y. Cáceres Escudero (UNEX 36532-1).

14 ejemplares ubicados a media ladera en mancha mixta de elevada heterogeneidad, formada por Arbutus unedo L., Viburnum tinus L., Quercus faginea Lam. subsp. broteroi (Cout.) A. Camus, $Q$. suber L., Q. pyrenaica Willd., Q. rotundifolia Lam., S. torminalis (L.) Crantz, Phillyrea angustifolia L., Erica arborea L. y Juniperus oxycedrus subsp. badia $(\mathrm{H}$. Gay) Debeaux.

ESPAÑA, Cáceres: Robledollano, Umbría del Manzano [30STJ8985], 570 m, 11.10.2012, Y. Cáceres Escudero (UNEX 36533-1). 2 ejemplares ubicados sobre derrubios de cuarcita en fondo de valle. Los individuos se sitúan en el interior de una mancha mixta de elevada heterogeneidad, formada por $A$. unedo, V. tinus, $Q$. faginea subsp. broteroi, $Q$. $x$ coutinhoi Samp., Q. rotundifolia, S. torminalis, E. arborea y $P$. angustifolia. En vaguadas aparece Ilex aquifolium L. y Prunus lusitanica subsp. Iusitanica L.

ESPAÑA, Cáceres: Cabañas del Castillo, Umbría del Aguazal [30STJ8984], 630 m, 08.11.2015, Y. Cáceres Escudero (UNEX 36531-1).

5 ejemplares ubicados en vaguada junto a $A$. unedo, $V$. tinus, $Q$. faginea subsp. broteroi y $S$. torminalis. La vaguada discurre a través de una zona de matorrales seriales de E. arborea y Cistus ladanifer subsp. ladanifer L.

ESPAÑA, Cáceres: Castañar de Ibor, Umbría del Camorro de Castañar [30STJ9288], $675 \mathrm{~m}$, 08.11.2015, Y. Cáceres Escudero (UNEX 36534-1).

1 ejemplar ubicado en el talud de un camino bajo el dosel arbóreo de un denso castañar de Castanea sativa Mill. Dicho ejemplar es el resultado del rebrote de cepa de un antiguo individuo que resultó gravemente afectado en un incendio ocurrido en 2005. Otras especies que comparten hábitat son $V$. tinus, S. torminalis, I. aquifolium y $P$. avium $\mathrm{L}$.

ESPAÑA, Cáceres: Alía, Sierra del Hospital del Obispo-Solana de Cervales [30SUJ0080], $1040 \mathrm{~m}$, 22.08.1992, M. Ladero \& J.M. Salinas (SALAF 25336). Robledal supramediterráneo. 1 ejemplar localizado en ecotono de robledal de $Q$. pyrenaica y quejigar de $Q$. faginea subsp. broteroi.

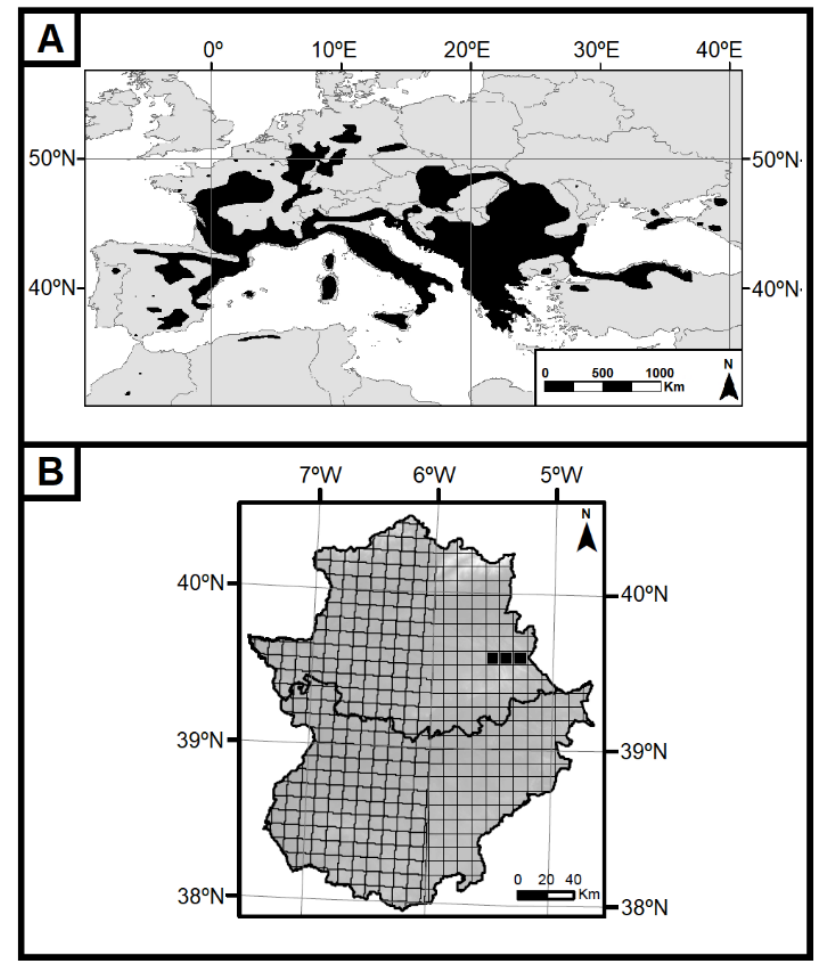

Figura 1. Mapas de distribución nativa de Sorbus domestica L.: A) rango global, B) distribución en Extremadura. El color negro representa la presencia de $S$. domestica (basado en cuadrículas UTM de 10×10 km).

Figure 1. Native distribution maps of Sorbus domestica L.: A) global range, B) distribution in Extremadura. Black colour represents $S$. domestica presence (based $10 \times 10$ km UTM grid cells).

\section{Distribución en Extremadura}

En Extremadura, S. domestica aparece representado por sólo 23 ejemplares, repartidos en 5 rodales que ocupan 3 cuadrículas UTM 10x10 que aglutinan cinco cuadrículas $1 \times 1 \mathrm{~km}$, dentro del macizo montañoso de las Villuercas, al sureste de la provincia de Cáceres (Figura 1B). Dos de las ubicaciones citadas en la bibliografía han sido confirmadas, mientras que las tres restantes han sido descubiertas en las prospecciones realizadas durante esta investigación (Tabla 1). Las principales características de su distribución son la elevada dispersión y fragmentación de los núcleos poblacionales, junto con el escaso número de ejemplares que los componen

La primera cita para $S$. domestica en el territorio extremeño fue dada a conocer por Ladero et al. (1993), señalando su existencia en al alto valle de río lbor (Figura 2A). Devesa (1995) recoge dicha referencia y la incorpora en el libro Flora $y$ Vegetación de Extremadura, citando la especie como muy rara y localmente naturalizada. Posteriormente, Oria de Rueda et al. (2006), en su trabajo sobre Botánica Forestal del Genero Sorbus en España, vuelve a mencionar a la especie en las lbores-Villuercas, añadiendo además, que se presenta muy escasa en las Hurdes y Sierra de Gata, dos localizaciones no encontradas en este 
estudio. Finalmente, durante los trabajos realizados bajo el proyecto FEA, y que derivaron en la realización del libro Los Bosques de Extremadura (Pulido et al., 2007), se localiza una nueva población de serbal, concretamente en el tramo medio del río Viejas (tributario del río lbor). Este trabajo reporta tres nuevos núcleos poblacionales en las Villuercas, por lo que, hasta la fecha, las poblaciones villuerquinas pueden considerarse como las únicas presentes en el territorio extremeño (Figura 1B).

En trabajos anteriores se había expuesto la posibilidad de que los ejemplares encontrados en las Villuercas se tratasen de individuos naturalizados
(Devesa, 1995), debido a que la especie ha sido cultivada en el pasado, tanto por sus frutos como por su madera (Oria de Rueda et al., 2006). Sin embargo, el hallazgo de nuevos ejemplares en las Villuercas, ubicados en masas forestales muy bien conservadas (Figura 2B y $2 \mathrm{C}$ ), alejadas de los núcleos de población y la existencia de otras localizaciones en los sistemas Oretano y Marianico (Lora \& Vivero, 1998; Rodríguez-Marzal \& PérezCarral, 2000; Perea \& Perea, 2008), refuerzan la naturalidad de la especie en la provincia corológica Luso-Extremadurense.
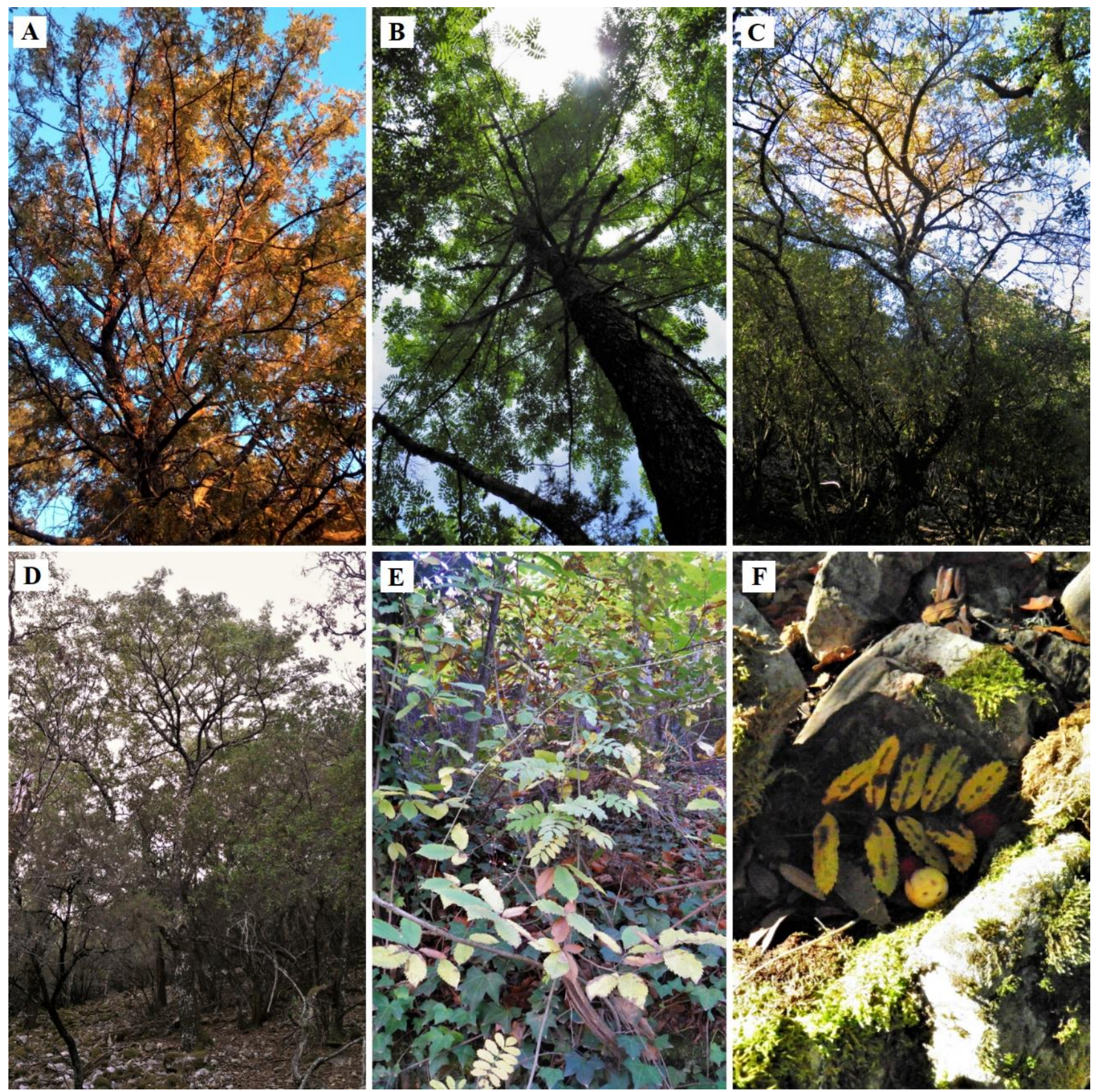

Figura 2. Ejemplares de Sorbus domestica L. pertenecientes a los distintos rodales existentes en Extremadura $(A=$ Solana de Cervales, $B=$ Umbría de la Venta, $C=$ Umbría del Aguazal, $D=$ Umbría del Manzano, $E=$ Umbría del Camorro de Castañar, $\mathrm{F}$ = fructificación de $\mathrm{S}$. domestica en el rodal de la Umbría del Aguazal).

Figure 2. Specimens of Sorbus domestica $L$. from the different patches in Extremadura $(A=$ Solana de Cervales, $B=$ Umbría de la Venta, $C=$ Umbría del Aguazal, $D=$ Umbría del Manzano, $E=$ Umbría del Camorro de Castañar, $F=$ fructification of S. domestica from the patch of Umbría del Aguazal). 


\section{Hábitat de la especie en Extremadura}

$S$. domestica medra en bosques muy bien conservados, participando de forma testimonial en el dosel arbóreo de ellos. El $91 \%$ de los individuos se localizan en el interior de manchas mixtas arbóreas de elevada heterogeneidad, pertenecientes a la asociación mesomediterránea Phillyreo angustifoliae - Arbutetum unedonis Rivas Goday \& Galiano in Rivas Goday, Borja, Esteve, Galiano, Rigual \& Rivas-Martínez 1960, aunque la especie también se ha localizado en el seno de castañares (fitosociológicamente adscritos a Quercenion roboripyrenaicae (Br.-BI., P. Silva \& Rozeira 1956) Rivas-
Martínez 1975) y en el ecotono de robledales (Sorb torminalis- Quercetum pyrenaicae (Rivas Goday 1964) Rivas-Martínez 1987) y quejigares (Pistacio terebinthi-Quercetum broteroi Rivas Goday in Rivas Goday, Borja, Esteve, Galiano, Rigual \& RivasMartínez 1960) (Rivas-Martínez et al., 1987). En cuanto a las principales características climáticas, la precipitación media anual de las ubicaciones donde se localizan las poblaciones de $S$. domestica es de $857,5 \pm 67,7 \mathrm{~mm}$, con una temperatura media anual de 13,6 $\pm 1,2{ }^{\circ} \mathrm{C}$, (Tabla 1), con un periodo de sequía en torno a los 2-3 meses (AEMET, 2011).

Tabla 1. Características generales de las poblaciones de Sorbus domestica L.

Table 1. General characteristics of the populations studied of Sorbus domestica $L$.

\begin{tabular}{|c|c|c|c|c|c|}
\hline POBLACIÓN & $\begin{array}{l}\text { Solana de } \\
\text { Cervales }\end{array}$ & $\begin{array}{c}\text { Umbría de la } \\
\text { Venta }\end{array}$ & $\begin{array}{l}\text { Umbría del } \\
\text { Aguazal }\end{array}$ & $\begin{array}{l}\text { Umbría del } \\
\text { Manzano }\end{array}$ & $\begin{array}{c}\text { Umbría del } \\
\text { Camorro de } \\
\text { Castañar }\end{array}$ \\
\hline $\begin{array}{c}\text { Referencia } \\
\text { bibliográfica }\end{array}$ & Ladero et al., 1993 & Inédita & Pulido et al., 2007 & Inédita & Inédita \\
\hline $\begin{array}{l}\text { Cuadrícula } \\
\text { UTM } 1 \times 1 \mathrm{~km}\end{array}$ & $30 S U J 0080$ & 30STJ8787 & 30STJ8984 & 30STJ8985 & 30STJ9288 \\
\hline $\begin{array}{l}\text { Rango } \\
\text { altitudinal } \\
\text { (msnm) }\end{array}$ & 1.040 & $655-699$ & $628-632$ & $568-575$ & 675 \\
\hline $\begin{array}{l}\text { Precipitación } \\
\text { media anual } \\
(\mathrm{mm})\end{array}$ & 976 & 808 & 842 & 840 & 820 \\
\hline $\begin{array}{c}\text { Temperatura } \\
\text { media anual } \\
\left({ }^{\circ} \mathrm{C}\right)\end{array}$ & 11 & 13,8 & 14,3 & 13,9 & 14,1 \\
\hline $\begin{array}{c}\text { Tamaño } \\
\text { poblacional }\end{array}$ & 1 & 14 & 5 & 2 & 1 \\
\hline $\begin{array}{c}\text { Piso } \\
\text { bioclimático } \\
\text { (Rivas- } \\
\text { Martínez, 1983) }\end{array}$ & $\begin{array}{l}\text { Supramediterráneo } \\
\text { inferior subhúmedo }\end{array}$ & $\begin{array}{l}\text { Mesomediterráneo } \\
\text { superior } \\
\text { subhúmedo }\end{array}$ & $\begin{array}{l}\text { Mesomediterráneo } \\
\text { medio subhúmedo }\end{array}$ & $\begin{array}{l}\text { Mesomediterráneo } \\
\text { superior } \\
\text { subhúmedo }\end{array}$ & $\begin{array}{l}\text { Mesomediterráneo } \\
\text { superior } \\
\text { subhúmedo }\end{array}$ \\
\hline $\begin{array}{l}\text { Comunidad } \\
\text { Fitosociológica } \\
\text { Sintáxón }\end{array}$ & $\begin{array}{c}\text { Pistacio terebinthi- } \\
\text { Quercetum } \\
\text { broteroi / Sorbo } \\
\text { torminalis- } \\
\text { Quercetum } \\
\text { pyrenaicae }\end{array}$ & $\begin{array}{c}\text { Phillyreo } \\
\text { angustifoliae - } \\
\text { Arbutetum } \\
\text { unedonis }\end{array}$ & $\begin{array}{c}\text { Phillyreo } \\
\text { angustifoliae - } \\
\text { Arbutetum } \\
\text { unedonis }\end{array}$ & $\begin{array}{c}\text { Phillyreo } \\
\text { angustifoliae- } \\
\text { Arbutetum } \\
\text { unedonis }\end{array}$ & $\begin{array}{c}\text { Quercenion robori- } \\
\text { pyrenaicae }\end{array}$ \\
\hline Litología & $\begin{array}{c}\text { Pizarras y } \\
\text { limonitas. Bloques } \\
\text { de cuarcita. }\end{array}$ & $\begin{array}{l}\text { Pizarras y } \\
\text { limonitas. }\end{array}$ & $\begin{array}{l}\text { Pizarras y } \\
\text { limonitas. }\end{array}$ & $\begin{array}{c}\text { Pizarras y } \\
\text { limonitas. Bloques } \\
\text { de cuarcita. }\end{array}$ & $\begin{array}{l}\text { Limonitas y } \\
\text { areniscas. }\end{array}$ \\
\hline $\begin{array}{c}\text { Tipo de suelo } \\
\text { (García- } \\
\text { Navarro, A. \& } \\
\text { López-Piñeiro, } \\
\text { 2002) }\end{array}$ & $\begin{array}{c}\text { Cambisol esquel - } \\
\text { epiléptico }\end{array}$ & $\begin{array}{c}\text { Acrisol } \\
\text { endoléptico }\end{array}$ & $\begin{array}{c}\text { Acrisol } \\
\text { endoléptico }\end{array}$ & $\begin{array}{c}\text { Acrisol } \\
\text { endoléptico }\end{array}$ & $\begin{array}{l}\text { Cambisol eutri - } \\
\text { endoléptico }\end{array}$ \\
\hline $\begin{array}{c}\text { pH del suelo } \\
(5 \mathrm{~cm}-20 \mathrm{~cm} \\
\text { de } \\
\text { profundidad })\end{array}$ & $6,3-6,5$ & $6,4-6,3$ & $5,9-5,7$ & $6,9-6,8$ & $6,1-5,9$ \\
\hline
\end{tabular}

La especie ocupa mayoritariamente situaciones de media ladera con orientación $\mathrm{N}-\mathrm{NE}$, en un rango altitudinal que oscila entre los 570 hasta los 1040 msnm, si bien el $96 \%$ de los ejemplares se localizan en el intervalo de 570 a $700 \mathrm{msnm}$. Al igual que otras ubicaciones encontradas de $S$. domestica en 
los sistemas Oretano y Mariánico (Lora \& Vivero, 1998; Rodríguez-Marzal \& Pérez-Carral, 2000; Perea \& Perea, 2008), el taxón se asienta sobre litologías de reacción ácida, tales como pizarras, limonitas, areniscas o cuarcitas (Tabla 1), en pendientes moderadas $(19,6 \pm 2,8 \%)$. La especie incluso ha sido localizada desarrollándose sobre gleras o derrubios de ladera de bloques de cuarcita (Figura 2D). Ladero et al. (1993) plantea que la existencia de capas de dolomías y calizas en el valle del Ibor (Villuercas), podría haber favorecido la presencia de la especie en esta zona. Oria de Rueda et al. (2006), recoge dicha hipótesis y señaló que la especie se encontraba silvestre en enclaves calizos de Los lbores, apunte erróneo y que ha sido refutado en este trabajo. Según el Mapa de Suelos de la Provincia de Cáceres (García-Navarro \& López-Piñeiro, 2002), los suelos sobre los que se desarrollan las poblaciones se engloban bajo la tipología de cambisoles y acrisoles (Tabla 1), inicialmente todos ellos con $\mathrm{pH}$ ácidos. Sin embargo, hay que denotar que $Q$. pyrenaica, especie forestal que acompaña a $S$. domestica en muchas de sus ubicaciones villuerquinas, parece que ejerce un bombeo de bases de capas profundas del suelo, consiguiendo que el $\mathrm{pH}$ de los horizontes edáficos superficiales se acerque a la neutralidad, como consecuencia del aporte de oxalato cálcico a través de la descomposición de las hojas caídas (RivasMartínez, 1964). En este sentido, los análisis del pH del suelo realizados en este estudio manifiestan esta tendencia, catalogándose como ligeramente ácidos o neutros y revelando que el $\mathrm{pH}$ de los horizontes edáficos superficiales es ligeramente más elevado que el de horizontes más profundos (Tabla 1).

\section{Reproducción, estado de conservación y amenazas}

La mayoría de los ejemplares localizados presentan un estado de conservación aceptable, observándose algunos daños causados por ungulados domésticos y silvestres, ya sea por ramoneo $o$ en menor medida, por escodaduras. Asimismo, los rebrotes vegetativos de raíz de la especie son copiosamente ramoneados, suprimiendo con ello la posible reproducción vegetativa. Otro de los daños observados ha sido el fuego, ya que afectó gravemente a un ejemplar en el año 2005, rebrotando satisfactoriamente tras el incendio (Figura 2E). Paralelamente, durante el periodo 2016-2019 se observó que el $65 \%$ de los ejemplares registrados florecieron y fructificaron (Figura 2F). Sin embargo, no se ha observado reclutamiento sexual alguno. Como consecuencia de esta nula reproducción sexual y vegetativa, todos los ejemplares detectados en Extremadura son adultos. La inspección visual de la estructura demográfica del total de ejemplares inventariados (Figura 3) se ajusta a un modelo demográfico con una asimetría negativa (Loetsch et al., 1973), denotando así una estructura demográfica envejecida, con muy pocos ejemplares juveniles y dominada por individuos de diámetros intermedios (Figura 3 ).

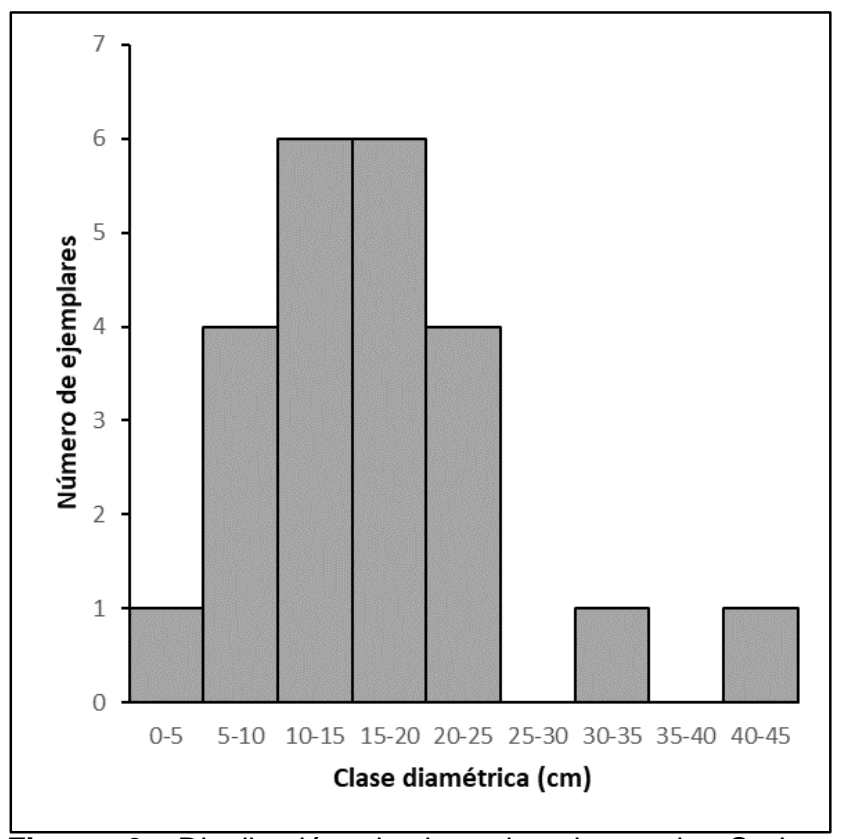

Figura 3. Distribución de los ejemplares de Sorbus domestica L. en Extremadura en función de las distintas categorías diamétricas.

Figure 3. Number of specimens of Sorbus domestica L. in Extremadura according to the different diametric categories.

A nivel europeo, se ha señalado la merma en la diversidad genética de $S$. domestica (George et al., 2015). Las poblaciones extremeñas podrían ser más proclives a dicha pérdida, fundamentalmente debido a dos aspectos; su escasa demografía y su dispersión poblacional, que conllevaría un exiguo o nulo flujo genético (Rotach, 2003).

Finalmente, debemos de señalar que $S$. domestica se encuentra catalogada como "Vulnerable" en el listado de especies del Catálogo Regional de Especies Amenazadas de Extremadura (DOE, 2018). Sin embargo, aplicando las prescripciones de la UICN (2012), el taxón en Extremadura se debería incluir en la categoría de "En Peligro Crítico" (CR), ya que cumple los criterios $\mathrm{B} 1 \mathrm{ab}$ (iii)+2ab(iii)+ $\mathrm{C} 2 \mathrm{a}$ (i)+D (área de ocupación de $0,1 \mathrm{~km}^{2}$, extensión de presencia de $39,34 \mathrm{~km}^{2}$ y tamaño de la población de 23 individuos maduros). Debido a ello, creemos que la administración regional debería de reevaluar la especie en un estatus de amenaza mayor, ya que un futuro próximo, se podría producir un colapso poblacional causado por la inexistente regeneración y la muerte por senectud de los ejemplares adultos. Además, la fragmentación poblacional, la dispersión de las poblaciones, el bajo número de ejemplares, junto con el escaso o nulo intercambio genético que caracterizan las poblaciones extremeñas, pueden incrementar los riesgos de extinción por estocasticidad ambiental, demográfica y genética (Menges, 1992; Hanski, 1998). Por lo tanto, medidas como la elaboración y puesta en marcha de planes de conservación, que conlleven la preservación de 
semillas en bancos de germoplasma, el reforzamiento de las poblaciones ya existentes y la creación de nuevas poblaciones con material genético procedente de los núcleos villuerquinos, serían medidas prioritarias necesarias para la protección de esta rara especie en Extremadura.

\section{Agradecimientos}

A Fernando Pulido y los técnicos que participaron en el desarrollo del proyecto FEA (Formaciones Forestales Autóctonas de Extremadura), por facilitar la ubicación de uno de los rodales estudiados. Al Herbario de la Universidad de Extremadura por su ayuda en las herborizaciones. A Carlos Arrate por facilitar el estudio de los ejemplares ubicados en su finca privada. Asimismo, agradecerle a la Agencia Estatal de Meteorología (AEMET), Delegación Territorial en Extremadura, por la cesión de las capas climáticas para la obtención de distintas variables.

\section{Bibliografía}

Aedo, C. \& Aldasoro, J.J. (1998). Sorbus. In F. Muñoz-Garmendia \& C. Navarro (Eds.), Flora Ibérica (Vol. VI Rosaceae, pp. 414-433). Madrid: Real Jardín Botánico de Madrid. CSIC.

AEMET (Agencia Estatal de Meteorología) (2011). Atlas climático ibérico. Madrid: Ministerio de Medio Ambiente y Medio Rural y Marino.

Calleja, J.A. \& Cáceres, Y. (2004). Vaccinium myrtillus L. y otras novedades corológicas para Extremadura y la comarca de Los Ibores-Las Villuercas. Studia Botanica, 23, 101-104.

Devesa, J.A. (1995). Vegetación y Flora de Extremadura. Badajoz: Universitas Editorial.

DOE (2018). Decreto 78 /2018, de 5 de junio, por el que se modifica el Decreto 37/2001, de 6 de marzo, que regula el Catálogo Regional de Especies Amenazadas de Extremadura.

García-Navarro, A. \& López-Piñeiro (2002). Mapa de suelos de la provincia de Cáceres. Escala 1:300.000. Badajoz: Universidad de Extremadura.

George, J.P., Konrad, H., Collin, E., Thevenet, J., Ballian, D., Idzojtic, M., Kamm, U., Zhelev, P. \& Geburek, T. (2015). High molecular diversity in the true service tree (Sorbus domestica) despite rareness: data from Europe with special reference to the Austrian occurrence. Annals of Botany, 115(7), 1105-1115. doi https://doi.org/10.1093/aob/mcv047

Hanski, I. (1998). Metapopulation dynamics. Nature, 396, 41-49. doi: https://doi.org/10.1038/23876
Ladero, M., Salinas, J.M. \& Santos, M.T. (1993). Dos nuevas plantas para la flora extremeña. Studia Botanica, 11, 297.

Loetsch, F., Zohrer, F. \& Haller, K.E. (1973). Forest inventory Volume II. Munich: BLV.

Lora, A. \& Vivero, J.L. (1998). Notas corológicas para la flora de Andalucía. Acta Botanica Malacitana, 23, 244.

Menges, E. (1992). Stochastic modeling of extinction in plant populations. In P.L. Fiedler \& S.K. Jain (Eds.), Conservation Biology: the theory and practice of nature conservation, preservation, and management (pp. 253-275). New york: Chapman \& Hall. doi: https://doi.org/10.1007/978-1-46846426-9_10.

Oria de Rueda, J.A., Martínez de Azagra, A. \& Álvarez, A. (2006). Botánica forestal del género Sorbus en España. Investigación Agraria. Sistemas y Recursos Forestales, 15, 166-186.

Perea, D.F. \& Perea, R. (2008). Vegetación y Flora de los Montes de Toledo: Guía de Campo. Toledo: Ediciones Covarrubias.

Philipps, J.B., Robertson, K.R., Smith, P.G., Rohrer, J.R. (1990). A checklist of the subfamily Maloideae (Rosaceae). Canadian Journal of Botany, 68 (10), 2209-2269. doi: https://doi.org/10.1139/b90-288

Pulido, F., Sanz, R., Abel, D., Ezquerra, J., Gil, A., González, G., Hernández, A., Moreno, G., Pérez. J.J. \& Vázquez, F.M. (2007). Los bosques de Extremadura. Evolución, Ecología y Conservación. Mérida: Junta de Extremadura. Consejeria de Industria.

Rivas-Martínez, S. (1964). Estudio de la vegetación y flora de las sierras de Guadarrama y Gredos. Anales Instituto del Botánico Cavanilles, 21 (1), 5-325.

Rivas-Martínez, S. (1983). Pisos bioclimáticos de España. Lazaroa, 5, 33-43.

Rivas-Martínez, Gandullo, J.M., Allué, J.L., Montero de Burgos. J.L. \& Gonzélez, J.L. (1987). Memoria del mapa de series de vegetación de España. Madrid: ICONA.

Rodríguez-Marzal, J.L. \& Pérez-Carral, M.C. (2000). Sobre la presencia de Sorbus domestica $L$. (Rosaceae) en Ciudad Real. Studia Botanica, 19, 109-112.

Rotach, P. (2003). EUFORGEN Technical Guidelines for genetic conservation and use for service tree (Sorbus domestica). Rome: International Plant Genetic Resources Institute.

UICN (2012). Categorías y Criterios de la Lista Roja de la UICN: Versión 3.1. Segunda edición. Gland y Cambridge: UICN. 